\title{
Using Amino Acids for Probing Structural Information of Cytochrome $c$ by Electrospray Ionization Mass Spectrometry
}

\author{
Haojie $\mathrm{Lu}^{*}$ and Yinlong Guo \\ Shanghai Mass Spectrometry Center, Shanghai Institute of Organic Chemistry, Chinese Academy of Sciences, \\ Shanghai, People's Republic of China
}

\section{Pengyuan Yang}

Research Center of Proteome and Department of Chemistry, Fudan University, Shanghai, People's Republic of China

\begin{abstract}
By using electrospray ionization mass spectrometry (ESI-MS), protein complexes of cytochrome $c$ with amino acids were studied. Different amino acids were investigated to explore these complexes. Using these amino acids, a strategy for probing the structure of cytochrome $c$ was established. It was found that L-Arg and L-Glu could bind with cytochrome $c$ to form noncovalent complexes. At low $\mathrm{pH}$ solution, complexes between the cytochrome $c$ molecule with several L-Arg molecules (multiple L-Arg adducts) were formed, and the number of binding ligands depended on the charge state of cytochrome $c$. While in neutral solution, the cytochrome $c$ molecule complexed with only one L-Arg molecule (single L-Arg adducts). As for L-Glu, only single L-Glu adducts were formed in both acidic and neutral solutions. (J Am Soc Mass Spectrom 2004, 15, 1612-1615) (c) 2004 American Society for Mass Spectrometry
\end{abstract}

$O^{2}$ ince the pioneering introduction and development of ESI-MS and matrix-assisted laser desorption/ ionization MS (MALDI-MS) by Fenn's group [1] and Karas's group [2], respectively, MS has been playing an increasingly important role in biological, biochemical, and biomedical research. With the most important characteristic of soft ionization, both ESI and MALDI allowed MS to be applied to analyze noncovalent complexes. Therefore, using ESI- and MALDI-MS, it has become possible to determine protein folding, characterize noncovalent protein complexes, and assess the contribution of an individual amino acid residue to a protein function.

The biological function of a protein depends directly on its noncovalent interactions with other components existing in the living system. Investigating these nonvovalent interactions is an important step to unraveling the mysteries of cellular function in health and disease states [3-5].

Over the last few years, the dramatically growing number of research articles studying the protein complexes by MS indicates that the instrument has become an important technology in this field because of its unrivaled speed, sensitivity, stoichiometry, and low sample con-

Published online September 16, 2004

Address reprint requests to Dr. Y. Guo, Shanghai Mass Spectrometry Center, Shanghai Institute of Organic Chemistry, Chinese Academy of Sciences, Shanghai 200032, People's Republic of China. E-mail: ylguo@mail.sioc.ac.cn

* Also at the Research Center of Proteome and Department of Chemistry, Fudan University, Shanghai 200032, People's Republic of China sumption [6-27]. MS has been used to probe the interactions of proteins with inhibitors, cofactors, metal ions, carbohydrates, other peptides and proteins, enzyme-substrate pairings, and nucleic acid complexes [6-27].

Previous studies have used acidic or basic small molecules to probe the basic or acidic amino acid residues of a wide variety of oligopeptides or proteins by counting the number of small molecules bound to the analytes [28-31]. However, using this method would reduce the ionization capability of the resulting complexes, which in turn may result in lower detection sensitivity by MS. Furthermore, the identification of these noncovalent complexes might become difficult due to the reducing of the number of ion charge states. These drawbacks can be solved by using amino acids as the probing small molecules. Although several protein/ peptide complexes have been studied before [32], to the best of our knowledge, protein/amino acid ligand complexes have never been reported. In this article, by using ESI-MS, we studied complexes of cytochrome $c$ with amino acid ligands.

\section{Experimental}

\section{Apparatus and Reagents}

The Mariner electrospray time-of-flight mass spectrometry (Applied Biosystems, Framingham, Boston, MA) was utilized in all MS experiments. A micro-electro- 
spray source was used. The ionspray voltage was 3800 $\mathrm{V}$ and the nozzle potential was $80 \mathrm{~V}$. The data were acquired by Mariner instrument control panel for positive ion mode from 100 to $4000 \mathrm{Da}$, and analyzed by Data Explorer software. The instrument was calibrated with PEG-800 solution. The samples were injected at a flow rate of $5 \mu \mathrm{l} / \mathrm{min}$ using a syringe pump.

Horse heart cytochrome $c$ was purchased from Sigma (St. Louis, MO). Amino acids were purchased from Shanghai Boao Institute of Science and Technology (People's Republic of China) as analytical pure reagents.

The noncovalent complexes were prepared by mixing cytochrome $c$ and amino acids solutions at a final concentration of $1.0 \times 10^{-4} \mathrm{M}$ for cytochrome $c$ and a range from $3.0 \times 10^{-4}$ to $3.0 \times 10^{-5} \mathrm{M}$ for amino acids.

\section{Results and Discussion}

\section{Optimization of the Solution Conditions}

Different added amounts of methanol to neutral mixtures solutions were investigated. We found that by using 15\% methanol, complexes of cytochrome $c$ with L-Arg produced the best sensitivity without significantly shifting the charge states (data not shown). Therefore, this percentage of methanol was used for all experiments. Next, we studied the effect of the L-Arg concentrations on the signal intensities of the resulted complexes. L-Arg concentrations range from $3.0 \times 10^{-4}$ to $3.0 \times 10^{-5} \mathrm{M}$ at a fixed cytochrome $c$ concentration of $1.0 \times 10^{-4} \mathrm{M}$ were investigated. Increasing the concentration of L-Arg up to $1.5 \times 10^{-4} \mathrm{M}$ resulted in increasing the intensities of these complexes due to the equilibrium of the noncovalent interaction. However, at L-Arg concentrations higher than $1.5 \times 10^{-4} \mathrm{M}$, the intensities of these complexes decreased because of the following possible two reasons: (1) The competition between these complexes and other compounds on protonation; (2) higher ionic strength which limited the ionizations of these complexes. Therefore, L-Arg concentration of $1.5 \times 10^{-4} \mathrm{M}$ was selected as the optimized one for all the experiments.

Because of its importance, the effect of changing the $\mathrm{pH}$ of the complexes solutions was also investigated. Accurate adjusting of the $\mathrm{pH}$ of these solutions was very difficult as no buffer was added. With and without the addition of acetic acid, the $\mathrm{pH}$ of these complex solutions were 2.5 and 5.5, respectively, and these two solutions were used for the further investigations.

\section{Characterization of Protein/Amino Acid Complexes}

Three groups of amino acids, including three basic amino acids: L-Arg, L-Lys and L-His, three neutral amino acids: L-Try, L-Tyr and L-Phe, and one acidic amino acid: L-Glu, were selected to probe the characteristics of cytochrome $c$ complexes at the optimized ESI and solution conditions. Among the studied amino

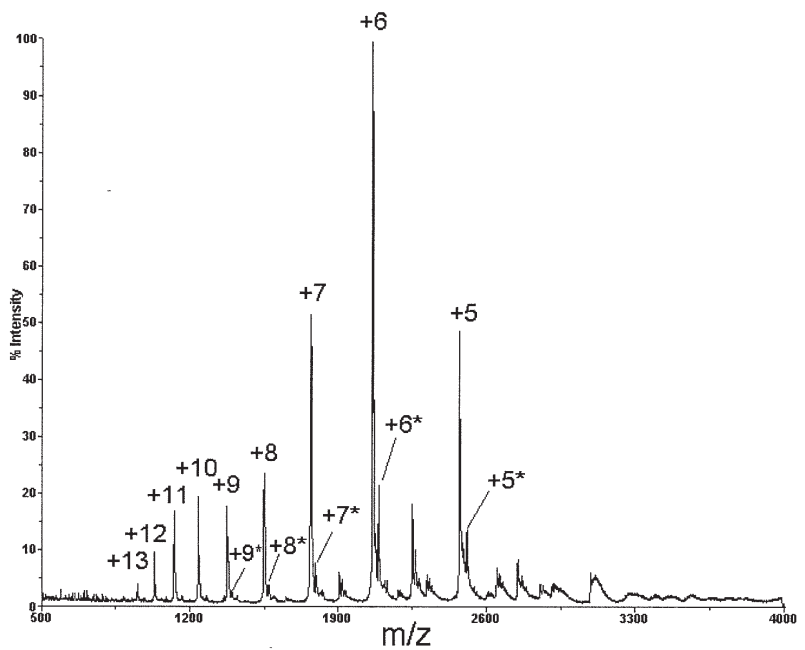

Figure 1. ESI mass spectrum of cytochrome $c\left(1.0 \times 10^{-4} \mathrm{M}\right)$ and L-Arg $\left(1.5 \times 10^{-4} \mathrm{M}\right)$ in $15 \%$ methanol water solution without adding acid ( $\mathrm{pH} 5.5)$.

acids, only L-Arg and L-Glu could form noncovalent complexes in solutions with $(\mathrm{pH}=2.5)$ and without $(\mathrm{pH}=5.5)$ the addition of acetic acid. While using other amino acids as probing molecules, noncovalent complexes were not formed in both solutions.

As shown in Figure 1, ESI of the cytochrome $c / \mathrm{L}$-Arg complexes at $\mathrm{pH} 5.5$ generated a distribution of charge states from +5 to +13 . Three distinct charge states $(+5$, +6 , and +7 ) gave the highest intensity signals. Only single L-Arg adducts could be identified at the charge states from +5 to +9 . The charge states distribution indicates that most cytochrome $c$ molecules adopted a folding conformation very close to the native conformation [32-36]. The spherical shape of the native conformation of cytochrome $c$ would limit the number of acidic amino acid residues available to interact with L-Arg. It should be noted that the other peaks that were produced by impurities were not assigned in Figure 1.

ESI of the cytochrome $c$ /L-Arg complexes at pH 2.5 generated a distribution of charge states from +6 to +18 , with the highest intensity at the charge state of +14 (Figure 2). Cytochrome $c$ contains 104 amino acid residues (including 12 acidic amino acids and 22 basic amino acids) with a covalently attached heme group. Below pH 4, cytochrome $c$ denatures [32-36], therefore, at $\mathrm{pH} 2.5$ cytochrome $c$ is expected to present a more extended conformation. For the charge states range from +10 to +12 , only single adducts were identified. However, multiple adducts were identified for charge states ranged from +6 to +9 . As the charge number increased, the number of bound L-Arg ligands decreased. At the high charge state of cytochrome $c$, it is most likely that the electrostatic repulsion limited the number of L-Arg ligands bound to the acidic amino acid residues. For the charge state of +6 , up to thirteen adducts (cytochrome $c$ molecule complexed with 13 L-Arg molecules) could be identified. This result indi- 


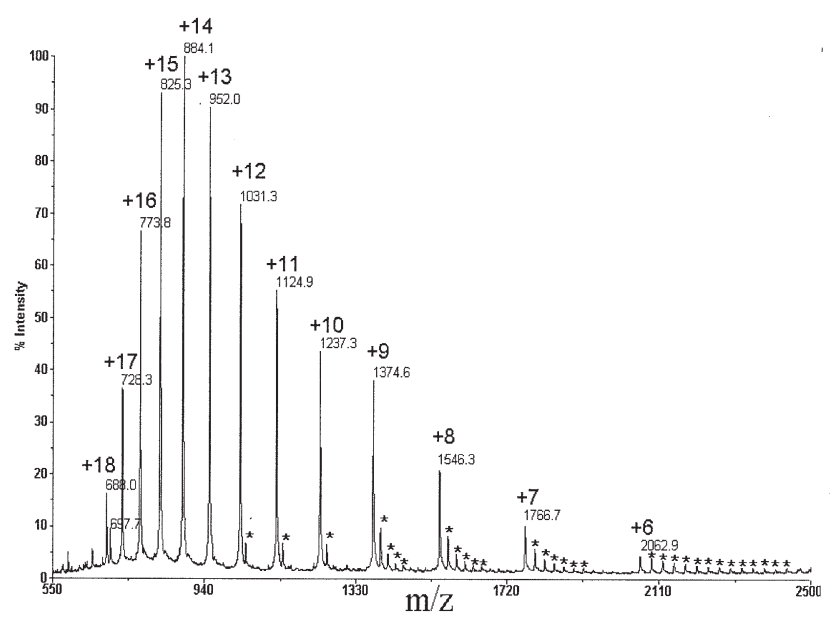

Figure 2. ESI mass spectrum of cytochrome $c\left(1.0 \times 10^{-4} \mathrm{M}\right)$ and L-Arg $\left(1.5 \times 10^{-4} \mathrm{M}\right)$ in $15 \%$ methanol water solution with adding acetic acid ( $\mathrm{pH} 2.5)$.

cates that there are totally 13 acidic binding sites in cytochrome $c$ available for interacting with the basic amino acid L-Arg. These 13 acidic binding sites are the 12 acidic amino acid resides and the C-terminal carboxyl group. So it can be concluded that cytochrome $c$ presents a fully extended conformation in the acidic solution ( $\mathrm{pH} 2.5)$.

Among the tested three basic amino acids of L-Arg, L-Lys, and L-His, only L-Arg could form noncovalent complexes with cytochrome $c$. This may be explained as L-Arg has a carbamidine analogical group that can form not only ionic interaction but also hydrogen bond at the interaction sites with cytochrome $c$. Besides its ionic interaction site, L-His can theoretically also form hydrogen bond, but the pentacycle may increase the steric repulsion and therefore, its amide protons would become unavailable. L-Lys has no ability to form hydrogen bond. Therefore, the ability of L-Arg to form hydrogen bond seems to be important to strengthen and stabilize its noncovalent complexes with cytochrome $c$. In addition, the gas phase basicities (GB) of the amino acids L-Arg, L-Lys, and L-His are 233.8, 226.0 , and $219.5 \mathrm{kcal} / \mathrm{mol}$, respectively [37]. It is expected that the higher GB value of an amino acid is, the stronger the ionic attraction force between these basic amino acids and the acidic amino acid resides in the cytochrome $c$.

The acidic amino acid probe L-Glu could also form complexes with cytochrome $c$. ESI of cytochrome $c / \mathrm{L}$ Glu complexes without acetic acid $(\mathrm{pH}=5.5)$ generated a distribution of charge states from +6 to +10 , with +8 giving the highest intensity. Only single L-Glu adducts were identified for charge states ranged from +6 to +8 . After the addition of acetic acid $(\mathrm{pH}=2.5)$, charge states shifted from lower to higher charge numbers, with +16 and +15 giving the highest intensity. However, similar to the results obtained at $\mathrm{pH} 5.5$, only single L-Glu adducts were formed at the charge states ranging from +6 to +8 . These results may be explained by the following. First, as we discussed earlier, only L-Arg has ionic as well as hydrogen bond interaction sites which make it able to form stable complexes with other amino acids. It is expected, therefore, that L-Arg residue as well would form stable complexes with L-Glu. Second, cytochrome $c$ has two Arg residues (Arg38 and Arg91). However, there are two acidic amino acid residues presented in both sides of Arg91 that may limit the binding of L-Glu to Arg91 residue due to the electrostatic repulsion. This might explain why only single L-Glu adducts were formed with cytochrome $c$ in both acidic and neutral solutions.

The three neutral amino acids L-Try, L-Tyr, and L-Phe failed to form any complex with cytochrome $c$ most probably due to the lack of the ionic attraction between the protein and these amino acids.

Considering the $\mathrm{pI}$ values of amino acids may also help to explain the reason why cytochrome $c$ formed complexes only with L-Arg and L-Glu. The $\mathrm{pI}$ values of the amino acids used in this study are as follows: Arg $=10.76$, His $=7.59$, Lys $=9.74$, Try $=5.89$, Tyr $=5.66$, Phe $=5.48$, and Glu $=3.22$. It is obvious that cytochrome $c$ formed complexes only with the two amino acids that have the highest and the lowest pI values among the studied group. These two amino acids, L-Arg and L-Glu, are expected to have strong ionic attractions with their partners of the amino acid residues of cytochrome $c$.

All together, our results indicate that to form a stable complex, there should be a strong ionic attraction and an ability to form a hydrogen bond between the amino acid residues of the protein and the amino acid probes. It should be mentioned that the complementing shapes between the protein and the amino acid probes could favor the formation of certain noncovalent complexes over the others.

\section{Acknowledgments}

The authors gratefully acknowledge support by the National Natural Science Foundation of China (no.20175034), and by the Chinese Academy of Sciences (no.00ZA14077).

\section{References}

1. Fenn, J. B.; Mann, M.; Meng, C. K.; Wong, S. F.; Whitehouse, C. M. Electrospray Ionization for the Mass Spectrometry of Large Biomolecules. Science 1989, 246, 64-71.

2. Karas, M.; Hillenkamp, F. 1Laser Desorption Ionization of Proteins with Molecular Masses Exceeding 10,000 Daltons. Anal. Chem. 1988, 60, 2299-2301.

3. Dobson, C. M. Protein Folding and Misfolding. Nature 2003, 18, 426(6968), 884-890.

4. Akiyama, S. K. Integrins in Cell Adhesion and Signaling. Hum. Cell 1996, 9(3), 181-186.

5. Pardee, A. B. Multiple Molecular Levels of Cell Cycle Regulation. J. Cell Biochem. 1994, 54(4), 375-378.

6. Tang, X.; Callahan, J. H.; Zhou, P.; Vertes, A. Noncovalent Protein-Oligonucleotide Interactions Monitored by Matrix- 
Assisted Laser Desorption/Ionization Mass Spectrometry. Anal. Chem. 1995, 67(24), 4542-4548.

7. Glocker, M. O.; Bauer, S. H.; Kast, J.; Volz, J.; Przybylski, M. Characterization of Specific Noncovalent Protein Complexes by UV Matrix-Assisted Laser Desorption Ionization Mass Spectrometry. J. Mass Spectrom. 1996, 31(11), 1221-1227.

8. Loo, J. A.; Holler, T. P.; Foltin, S. K.; McConnell, P.; Banotai, C. A.; Horne, N. M.; Mueller, W. T.; Stevenson, T. I.; Mack, D. P. Application of Electrospray Ionization Mass Spectrometry for Studying Human Immunodeficiency Virus Protein Complexes. Proteins 1998, 2, 28-37.

9. Raftery, M. J.; Geczy, C. L. Identification of Noncovalent Dimeric Complexes of the Recombinant Murine S100 Protein CP10 by Electrospray Ionization Mass Spectrometry and Chemical Cross-Linking. J. Am. Soc. Mass Spectrom. 1998, 9(5), 533-539.

10. Veenstra, T. D.; Tomlinson, A. J.; Benson, L.; Kumar, R.; Naylor, S. Low Temperature Aqueous Electrospray Ionization Mass Spectrometry of Noncovalent Complexes. J. Am. Soc. Mass Spectrom. 1998, 9(6), 580-584.

11. Xu, N.; Pasa-Tolic, L.; Smith, R. D.; Ni, S.; Thrall, B. D. Electrospray Ionization-Mass Spectrometry Study of the Interaction of Cisplatin-Adducted Oligonucleotides with Human XPA Minimal Binding Domain Protein. Anal. Biochem. 1999, 15, 272(1), 26-33.

12. Borchers, C.; Tomer, K. B. Characterization of the Noncovalent Complex of Human Immunodeficiency Virus Glycoprotein 120 with its Cellular Receptor CD4 by Matrix-Assisted Laser Desorption/Ionization Mass Spectrometry. Biochemistry 1999, 38(36), 11734-11740.

13. Vogl, T.; Roth, J.; Sorg, C.; Hillenkamp, F.; Strupat, K. Calcium-Induced Noncovalently Linked Tetramers of MRP8 and MRP14 Detected by Ultraviolet Matrix-Assisted Laser Desorption/Ionization Mass Spectrometry. J. Am. Soc. Mass Spectrom. 1999, 10(11), 1124-1130.

14. Kiselar, J. G.; Downard, K. M. Reservation and Detection of Specific Antibody-Peptide Complexes by Matrix-Assisted Laser Desorption Ionization Mass Spectrometry. J. Am. Soc. Mass Spectrom. 2000, 11(8), 746-750.

15. Freitas, M. A.; Hendrickson, C. L.; Marshall, A. G.; Rostom, A. A.; Robinson, C. V. Competitive Binding to the Oligopeptide Binding Protein, OppA: In-Trap Cleanup in an Fourier Transform Ion Cyclotron Resonance Mass Spectrometer. J. Am. Soc. Mass Spectrom. 2000, 11(11), 1023-1026.

16. Horn, D. M.; Breuker, K.; Frank, A. J.; McLafferty, F. W. Kinetic Intermediates in the Folding of Gaseous Protein Ions Characterized by Electron Capture Dissociation Mass Spectrometry. J. Am. Chem Soc. 2001, 123(40), 9792-9799.

17. Bacher, G.; Szymanski, W. W.; Kaufman, S. L.; Zollner, P.; Blaas, D.; Allmaier, G. Charge-Reduced Nano Electrospray Ionization Combined with Differential Mobility Analysis of Peptides, Proteins, Glycoproteins, Noncovalent Protein Complexes and Viruses. J. Mass Spectrom. 2001, 36(9), 1038-1052.

18. de Brouwer, A. P.; Versluis, C.; Westerman, J.; Roelofsen, B.; Heck, A. J.; Wirtz, K. W. Determination of the Stability of the Noncovalent Phospholipid Transfer Protein-Lipid Complex by Electrospray Time-of-Flight Mass Spectrometry. Biochemistry 2002, 41(25), 8013-8018.

19. van den Bremer, E. T.; Jiskoot, W.; James, R.; Moore, G. R.; Kleanthous, C.; Heck, A. J.; Maier, C. S. Probing Metal Ion Binding and Conformational Properties of the Colicin E9 Endonuclease by Electrospray Ionization Time-of-Flight Mass Spectrometry. Protein Sci. 2002, 11(7), 1738-1752.

20. Gabelica, V.; Vreuls, C.; Filee, P.; Duval, V.; Joris, B.; Pauw, E. D. Advantages and Drawbacks of Nanospray for Studying
Noncovalent Protein-DNA Complexes by Mass Spectrometry. Rapid Commun. Mass Spectrom. 2002, 16(18), 1723-1728.

21. Clark, S. M.; Konermann, L. Diffusion Measurements by Electrospray Mass Spectrometry for Studying Solution-Phase Noncovalent Interactions. J. Am. Soc. Mass Spectrom. 2003, 14(5), 430-441.

22. Woods, A. S.; Fuhrer, K.; Gonin, M.; Egan, T.; Ugarov, M.; Gillig, K. J.; Schultz, J. A. Angiotensin II-Acetylcholine Noncovalent Complexes Analyzed with MALDI-Ion Mobility-TOF MS. J. Biomol. Tech. 2003, 14(1), 1-8.

23. Benesch, J. L.; Sobott, F.; Robinson, C. V. Thermal Dissociation of Multimeric Protein Complexes by Using Nanoelectrospray Mass Spectrometry. Anal. Chem. 2003, 15, 75(10), 2208-2214.

24. Kowalski, P.; Suder, P.; Kowalska, T.; Silberring, J.; Duszynska, B.; Bojarski, A. J. Electrospray Mass Spectrometric Studies of Noncovalent Complexes of Buspirone Hydrochloride and Other Serotonin 5-HT(1A) Receptor Ligands Containing Arylpiperazine Moieties. Rapid Commun. Mass Spectrom. 2003, 7(18), 2139-2146.

25. Hagan, N.; Fabris, D. Direct Mass Spectrometric Determination of the Stoichiometry and Binding Affinity of the Complexes Between Nucleocapsid Protein and RNA Stem-Loop Hairpins of the HIV-1 PSI-Recognition Element. Biochemistry 2003, 16, 42(36), 10736-10745.

26. Zhang, S.; Van Pelt, C. K.; Wilson, D. B. Quantitative Determination of Noncovalent Binding Interactions Using Automated Nanoelectrospray Mass Spectrometry. Anal. Chem. 2003, 75(13), 3010-3018.

27. Wendt, S.; McCombie, G.; Daniel, J.; Kienhofer, A.; Hilvert, D.; Zenobi, R. Quantitative Evaluation of Noncovalent Chorismate Mutase-Inhibitor Binding by ESI-MS. J. Am. Soc. Mass Spectrom. 2003, 14(12), 1470-1476.

28. Stephenson, J. L.; McLuckey, S. A. Counting Basic Sites in Oligopeptides via Gas-Phase Ion Chemistry. Anal. Chem. 1997, 69, 281-285.

29. McLuckey, S. A.; Wells, J. M.; Stephenson, J. L.; Goeringer, D. E. Novel Quadropole Ion Trap Methods for Characterizing the Chemistry of Gaseous Macro-Ions. Int. J. Mass Spectrom. 2000, 200, 137-161.

30. Friess, S. D.; Zenobi, R. Protein Structure Information from Mass Spectrometry? Selective Titration of Arginine Residues by Sulfonates. J. Am. Soc. Mass Spectrom. 2001, 12, 810-818.

31. Friess, S. D.; Daniel, J. M.; Hartmann, R.; Zenobi, R. Mass Spectrometric Noncovalent Probing of Amino Acids in Peptides and Proteins. Int. J. Mass Spectrom. 2002, 219, 269-281.

32. Schnier, P. D.; Gross, D. S.; Williams, E. R. Electrostatic Forces and Dielectric Polarizability of Multiply Protonated Gas-Phase Cytochrome $c$ Ions Probed by Ion/Molecule Chemistry. J. Am. Chem. Soc. 1995, 117, 6747-6757.

33. McLuckey, S. A.; Van Berkel, G. J.; Glish, G. L. Reactions of Dimethylamine with Multiply Charged Ions of cytochrome $c$. J. Am. Chem. Soc. 1990, 112, 5668-5670.

34. Wagner, D. S.; Anderegg, R. J. Conformation of Cytochrome $c$ Studied by Deuterium Exchange-Electrospray Ionization Mass Spectrometry. Anal. Chem. 1994, 66, 706-711.

35. Shelimov, K. B.; Clemmer, D. E.; Hudgins, R. R.; Jarrold, M. F. Protein Structure in VacuoGas-Phase Conformations of BPTI and Cytochrome c. J. Am. Chem. Soc. 1997, 119, 2240-2248.

36. Gong, S.; Camara, E.; He, F.; Green, M. K.; Lebrilla, C. B. Chiral Recognition and the Deprotonation Reaction of Gas-Phase Cytochrome c Ions. Int. J. Mass Spectrom. 1999, 185/186/187, 401-412.

37. Gorman, G. S.; Speir, J. P.; Turner, C. A.; Amster, I. J. Proton Affinities of the 20 Common $\alpha$-Amino Acids. J. Am. Chem. Soc. 1992, 114, 3986-3988. 